\title{
Comparison of Experimental and Calculated Peak Shapes for Three Cylindrical Geometry FAIMS Prototypes of Differing Electrode Diameters
}

\author{
Roger Guevremont and Randy Purves \\ Ionalytics Corporation, Ottawa, Ontario, Canada
}

\begin{abstract}
High-field asymmetric waveform ion mobility spectrometry (FAIMS) separates ions at atmospheric pressure and room temperature based on the difference of the mobility of ions in strong electric fields and weak electric fields. This field-dependent mobility of an ion is reflected in the compensation voltage (CV) at which the ion is transmitted through FAIMS, at a given asymmetric waveform dispersion voltage (DV). Experimental CV, relative peak ion intensity, and peak width data were compared for three FAIMS prototypes with concentric cylindrical electrodes having inner/outer electrode radii of: (1) $0.4 / 0.6 \mathrm{~cm},(2) 0.8 / 1.0 \mathrm{~cm}$, and (3) $1.2 / 1.4 \mathrm{~cm}$. The annular analyzer space was $0.2 \mathrm{~cm}$ wide in each case. A finite-difference numerical computation method is described for evaluation of peak shapes and widths in a CV spectrum collected using cylindrical geometry FAIMS devices. Simulation of the radial distribution of the ion density in the FAIMS analyzer is based upon calculation of diffusion, electric fields, and the electric fields introduced by coulombic ion-ion repulsion. Excellent agreement between experimental and calculated peak shapes were obtained for electrodes of wide diameter and for ions transmitted at low CV. (J Am Soc Mass Spectrom 2005, 16, 349-362) ( 2004 American Society for Mass Spectrometry
\end{abstract}

$\mathrm{H}$ igh-field asymmetric waveform ion mobility spectrometry (FAIMS) is a technology that separates ions at atmospheric pressure and room temperature based on the difference in the mobility of an ion in strong and weak electric fields [1-4]. The Guevremont research group [4] presented the first description of the unique ion focusing properties of a cylindrical geometry FAIMS. A second report [5] further refined the description of the ion focusing mechanism, and provided the first experimental measurement of the (nonuniform) radial distribution of the ions between the concentric cylinders. By taking advantage of the ion focusing in a cylindrical geometry FAIMS, an atmospheric pressure ion trap was designed and tested $[6,7]$. Ion half-lives in excess of $2 \mathrm{~s}$ were demonstrated [8]. Ion trajectory modeling provided insight into the virtual potential well that formed in cylindrical geometry FAIMS [7].

FAIMS has been shown to be a powerful ion processing tool for improving the detection limits of quantitative analysis using mass spectrometry, particularly

Published online January 19, 2005

Address reprint requests to Dr. R. Guevremont, Ionalytics Corporation, Bldg. M50-IPF, 1200 Montreal Road, Ottawa, Ontario K1A 0R6, Canada. E-mail: roger.guevremont@ionalytics.com for ion sources characterized by a high intensity of background ions. Analytical applications ranging from detection of inorganic species to the study of protein conformers have been demonstrated [9-16].

In a recent paper, Smith and co-workers have undertaken a study of the resolution and sensitivity of FAIMS [17], making comparisons between experimental data and the results of ion trajectory computations. Shvartsburg et al. [18] have also studied the behavior of several ions in gas mixtures, and compared experimental results with predictions based on the theory of ion mobility in mixtures of gases [19]. The theory and newly proposed models (unique to FAIMS) for mobility changes in gas mixtures based on the formation and dissociation of labile complexes [20,21] are discussed [18]. More recently, Smith and co-workers studied the impact of the design and the optimization of the operation of FAIMS analyzers [22]. Several experimental parameters, including the width of the analytical gap, waveform frequency and profile, and gas flow speed were investigated.

Application of FAIMS as an ion processing tool for mass spectrometry may require electrodes with performance characteristics uniquely tuned for solving disparate analytical problems. For example, the separation of conformers of proteins and the separation of isomers 


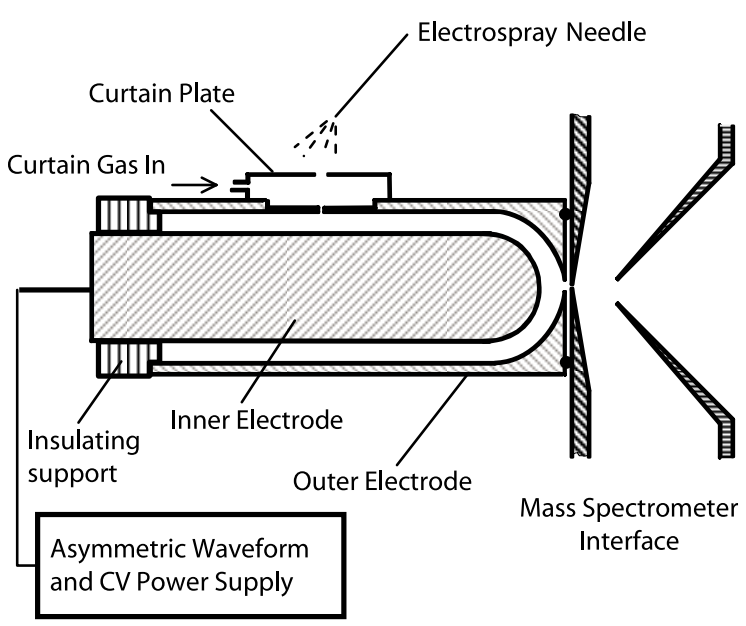

Figure 1. Simplified schematic of FAIMS electrodes based on cylindrical inner and outer electrode radii terminated in a hemisphere. Three electrodes of this geometry were evaluated with inner/outer electrodes of $0.4 / 0.6 \mathrm{~cm}, 0.8 / 1.0 \mathrm{~cm}$, and $1.2 / 1.4 \mathrm{~cm}$, respectively. The electrodes were mounted in gas-tight contact with the orifice plate of the mass spectrometer.

may require optimized resolution, whereas the analysis of tryptic fragments of a protein digest may be better served by a device capable of high sensitivity, high signal-to-background ratio, but having sufficiently low resolution that a maximum number of multiply charged ions can be detected without stepping CV [23]. The diameter of the electrodes in FAIMS is a key mechanical parameter controlling the resolution and sensitivity of FAIMS. In this report, the relationship between the diameter of cylindrical electrodes and the peak width in the CV scan taken with FAIMS is considered. Three FAIMS prototypes were constructed having different radii of the inner and outer electrodes. The design and operation of these prototypes is described, and the peak shapes and widths in the $\mathrm{CV}$ spectra using these devices are compared. A finite-difference numerical simulation of ion distribution in the FAIMS analyzer is described that includes the effects of electric fields, diffusion, and space charge. The peak shape and peak widths of calculated and experimentally determined peaks using three diameters of cylindrical geometry FAIMS are compared.

\section{Experimental}

Figure 1 illustrates a domed version of FAIMS electrodes in which the inner cylindrical electrode is terminated as a hemisphere. For this study, three diameters of FAIMS domed electrodes were designed and fabricated to permit an evaluation of the effect of cylinder radii on the peak shapes and peak widths in the FAIMS spectra. The inner cylinders had radii of $0.4,0.8$, and $1.2 \mathrm{~cm}$, and the outer cylinders of each prototype had radii that were $0.2 \mathrm{~cm}$ larger. The ions were separated in the $0.2 \mathrm{~cm}$ wide annular space between these concentric cylinders. The distance from the entrance orifice, through which the ions entered the analyzer region, to the tip of the hemisphere was about $3.5 \mathrm{~cm}$. A gas flow of $0.5 \mathrm{~L} / \mathrm{min}$ carried the ions along the analyzer region. The minimum time required for the ions to travel the length of the analyzer was measured and determined to be about $170 \mathrm{~ms}$ (electrodes with inner/outer radii of $0.8 / 1.0 \mathrm{~cm}$ ). The time required for the measured signal to stabilize was significantly longer (over $500 \mathrm{~ms}$ ), and indicates that the ion/gas mixtures may take diversely different pathways between the inlet and the outlet of FAIMS.

As shown in Figure 1, the inner cylinder of FAIMS is terminated as a hemisphere with the same radius as the cylindrical portion of the electrode. The end of the outer cylinder is also hemispherical in shape, and has an opening of about $1 \mathrm{~mm}$ at its center, with razor sharp edges, that serves as an exit aperture for the ion stream. In the FAIMS device shown in Figure 1, provision has been made for adjustment of the longitudinal location of the inner cylinder. In practice, a space of between 0.17 and $0.25 \mathrm{~cm}$ between the end of the inner electrode and the exit port in the outer electrode is required for optimum operation of FAIMS. Since the surfaces at the end of the inner electrode are curved in a third dimension relative to the cylindrical portion of the inner electrode, the electric fields are not identical to those along the annular analyzer region. For this reason, the longitudinal location of the inner electrode was made to be adjustable and the optimum location was established empirically. The radial variation in electric field strength at the hemispherical end of the inner electrode differs slightly from the radial variation of fields along the length of the cylindrical portion of FAIMS; both have been modeled with numerical processing techniques [7]. Modeling shows that the resolution of the spherical region is lower than that of the cylindrical portion of the analyzer, therefore there exists a range of longitudinal locations of the inner electrode that do not impact on the peak shape. The impact of the longitudinal location of the inner electrode on the resolution of FAIMS is the subject of an ongoing investigation and beyond the scope of this report.

For this study, the ions were generated by electrospray ionization. A fine tipped capillary was held at about $-2000 \mathrm{~V}$ relative to the FAIMS curtain plate. A solution of $1 \mathrm{ppm}$ bromochloroacetic acid in 9/1 $\mathrm{MeOH} /$ water containing $0.2 \mathrm{mM} \mathrm{NH} \mathrm{NH}_{4} \mathrm{OAc}$ was delivered at a flow rate of $1 \mu \mathrm{L} / \mathrm{min}$ to the electrospray capillary by a syringe pump. The FAIMS curtain plate was held about $1000 \mathrm{~V}$ more negative than the outer FAIMS electrode $(-8 \mathrm{~V})$ to assist in ion transmission across the desolvation region. A flow of purified, dry, nitrogen curtain gas $(2.5 \mathrm{~L} / \mathrm{min})$ was necessary to help desolvate the ions produced by electrospray, and to minimize the entrance of droplets and neutral molecules of the solvents into the FAIMS analyzer. Ion separation and transmission through FAIMS is adversely affected by trace contaminants in the carrier gas stream [4]. 
The transmission of bromochloroacetate anion $\left(\mathrm{BCA}^{-}\right)$through each FAIMS prototype was established by application of an asymmetric waveform [24] with a range of dispersion voltages from $\mathrm{DV}=-1800$ to $-3960 \mathrm{~V}$, and a scan of CV from 0 to $+25 \mathrm{~V}$. Mass spectrometric ion-selective compensation voltage (IS$\mathrm{CV})$ scans for $\mathrm{BCA}^{-}(\mathrm{m} / z-173)$ were used to establish the optimum $\mathrm{CV}$ of transmission through FAIMS. A PE-Sciex API 300 triple quadrupole mass spectrometer was used to detect the ions transmitted through FAIMS. The mass spectrometer operating conditions were determined during earlier experiments [9] with the haloacetic acids. The original curtain plate of the PE-SCIEX API 300 was removed to gain access to the orifice plate, which was modified to permit relatively fast exchange of the FAIMS prototypes. The FAIMS prototypes were all constructed with a PEEK ring designed to be mounted onto the instrument using holes threaded into the orifice plate. The outer cylinders of the FAIMS prototypes were inserted into the PEEK sleeve until the outer cylinder was in contact with the orifice plate of the mass spectrometer. No additional modification of the instrument was necessary, and exchange of prototypes did not require venting of the instrument. Gas flow through the FAIMS prototypes was controlled by the rate of flow of gas through the orifice leading into the vacuum chamber of the mass spectrometer. The orifice in the PE-Sciex API 300 instrument is about $100 \mu \mathrm{m}$ diameter, and the flow into this orifice was determined to be about $0.5 \mathrm{~L} / \mathrm{min}$. The FAIMS prototypes were mounted and sealed against the orifice plate of the instrument so that the gas flow through the analyzer region was equal to the gas flow into the vacuum chamber. Experiments indicated that this mode of operation resulted in maximization of ion transmission efficiency between FAIMS and the mass spectrometer. The OR voltage of the PE-Sciex API 300 was used to provide the FAIMS compensation voltage. In this way the scans of the FAIMS compensation voltage were convenient and compatible with the data acquisition system (using LCTune). For this work, CV spectra were acquired from 0 to $+25 \mathrm{~V}$ with a step size of $0.1 \mathrm{~V}$.

\section{Results and Discussion}

\section{Transmission of Bromochloroacetate Anion at High Dispersion Voltage}

Figure 2a shows a series of experimental IS-CV scans for $\mathrm{BCA}^{-}$using FAIMS having inner and outer electrode radii of 0.8 and $1.0 \mathrm{~cm}$ respectively, at dispersion voltages from -1800 to $-3960 \mathrm{~V}$. Three changes in the transmission of this ion are observed as the DV increased. First, the CV of the optimum transmission increased with applied DV, and secondly, the efficiency of the ion transmission increased significantly and finally the width of the peak increased. The increase of transmission is a consequence of the ion focusing in this cylindrical geometry FAIMS [5]. The increase of mea-
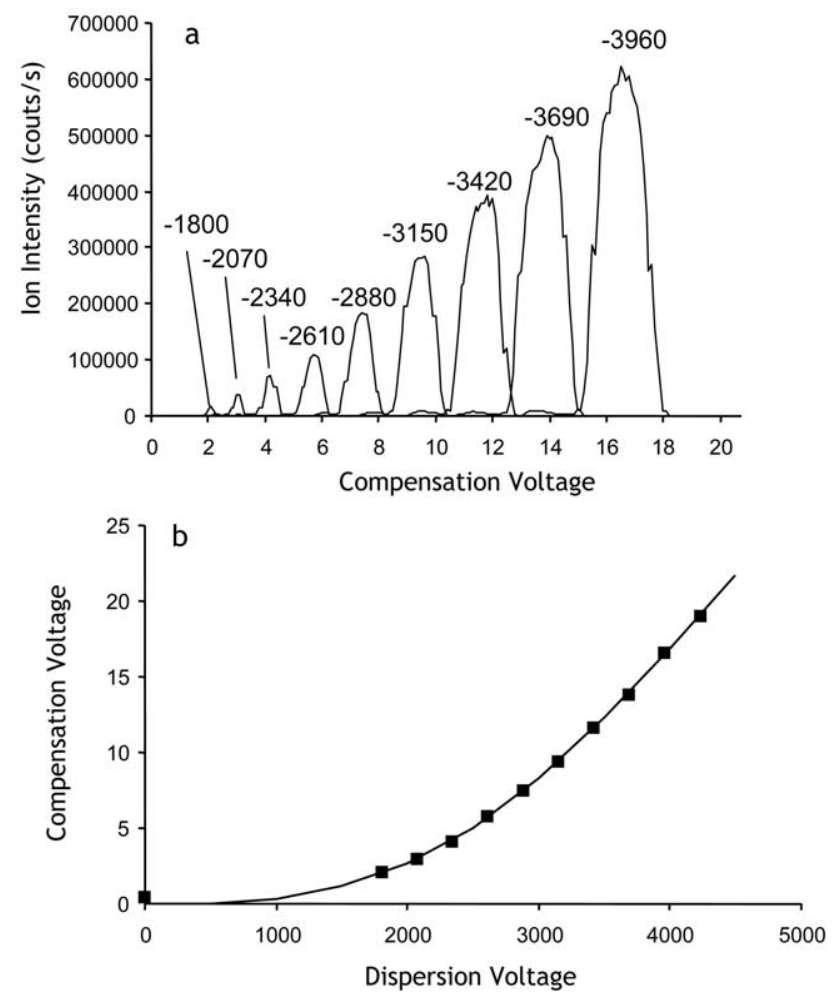

Figure 2. (a) Experimental CV scans taken while monitoring $\mathrm{m} / \mathrm{z}$ $-173\left(\mathrm{BCA}^{-}\right)$. The scans were collected at a series of applied waveforms from DV $=-1800$ to $\mathrm{DV}=-3960 \mathrm{~V}$ using FAIMS electrodes with radii of $0.8 / 1.0 \mathrm{~cm}$. (b) The CV of optimum transmission at each experimental DV (markers) and expected CV of transmission (solid line) calculated using high field parameters $\alpha=7.984 \mathrm{E}-6 \mathrm{Td}^{-2}$ and $\beta=-3.049 \mathrm{E}-10 \mathrm{Td}^{-4}$.

sured $\mathrm{CV}$ reflects changes in the difference between the ion mobility at high electric field relative to the ion mobility at low electric field. The markers in Figure $2 b$ are the experimental CV values for the series of applied waveforms with DV values from -1800 to $-4250 \mathrm{~V}$. The solid curve corresponds to the calculated values of expected $\mathrm{CV}$ at each DV using the parameters $\alpha=$ 7.984E- $6 \mathrm{Td}^{-2}$ and $\beta=-3.049 \mathrm{E}-10 \mathrm{Td}^{-4}$, which are used to describe the behavior of the ion mobility at high electric field strength in:

$$
K_{h}=K\left(1+\alpha(E / N)^{2}+\beta(E / N)^{4}\right)
$$

The parameters $\alpha$ and $\beta$ were determined from the experimental values of DV and CV (markers in Figure 2b). If FAIMS is operated by application of an asymmetric waveform described by:

$$
V_{a}(t)=C_{v}+D \frac{2}{3}{ }_{\nu} \sin (\omega t)+\frac{1}{3} D_{\nu} \sin \left(2 \omega t-\frac{\pi}{2}\right)
$$

where $D_{v}$ is the dispersion voltage, $C_{v}$ is the compensation voltage, and $\omega$ is the waveform frequency (radians/s), the experimental values of $C_{v}$ and $D_{v}$ for a series of measurements taken at several values of applied dispersion voltage can be used in a regression analysis to determine $\alpha$ and $\beta$ using eq 3 [24-26]. 


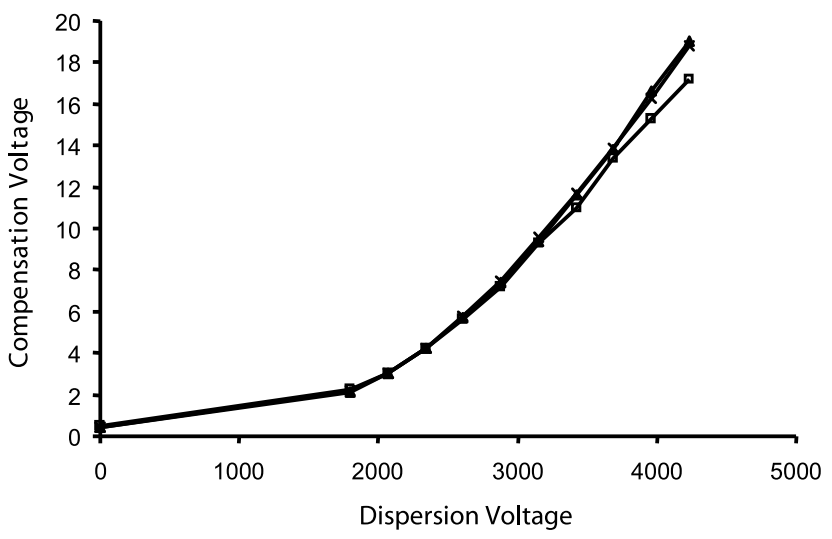

Figure 3. Optimum experimentally observed $\mathrm{CV}$ of transmission of $\mathrm{m} / z-173\left(\mathrm{BCA}^{-}\right)$collected at $\mathrm{DV}=-1800$ to $\mathrm{DV}=-4230 \mathrm{~V}$ using FAIMS prototypes having inner/outer electrode radii of $0.4 / 0.6 \mathrm{~cm}$ (open square), $0.8 / 1.0 \mathrm{~cm}(\mathrm{X})$, and $1.2 / 1.4 \mathrm{~cm}$ (open triangle).

$$
\begin{aligned}
& C_{v}+\alpha\left(\frac{D_{v}^{3}}{9 d^{2}}+\frac{15 C_{v} D_{v}^{2}}{18 d^{2}}+\frac{C_{v}^{3}}{d^{2}}\right) \\
& +\beta\left(\frac{55 D_{v}^{5}}{486 d^{4}}+\frac{55 C_{\nu} D_{v}^{4}}{72 d^{4}}+\frac{10 C_{v}^{2} D_{v}^{3}}{9 d^{4}}+\frac{25 C_{v}^{3} D_{v}^{2}}{9 d^{4}}+\frac{C_{v}^{5}}{d^{4}}\right) \\
& =0
\end{aligned}
$$

Here, $d$ is the width of the analyzer region $(\mathrm{cm})$. Many of the terms in eq 3 are small, and a simplified version of this equation has been used previously [27, 28]. Care must be taken in using this equation; a tutorial can be found on the Web [29].

\section{Comparison of the Transmission of $B C A^{-}$ in Three FAIMS Prototypes of Different Electrode Diameters}

Two comparisons of the transmission of the $\mathrm{BCA}^{-}$ through FAIMS will be considered. First, the CV of optimum transmission will be evaluated for each of the FAIMS prototypes of differing electrode diameters. Second, the peak shapes and peak widths will be determined for each of these devices.

Figure 3 illustrates the experimental CV of optimum transmission of $\mathrm{BCA}^{-}$through the three FAIMS prototypes (inner electrodes having radii of 0.4, 0.8, and $1.2 \mathrm{~cm}$, respectively) as a function of the applied DV. As expected, there is only a slight difference in the optimum CV experimentally observed using each of the three prototypes. The results for the two wider diameter FAIMS were in excellent agreement. The peak shapes in the CV spectra at high DV and with the narrowest electrodes (inner/outer radii, $0.4 / 0.6 \mathrm{~cm}$ ) were distorted, and there is a shift of $\mathrm{CV}$.

Figure 4 shows $C V$ scans taken while monitoring $\mathrm{m} / \mathrm{z}$ -173 of $\mathrm{BCA}^{-}$(intensities normalized) for the three FAIMS prototypes with electrode radii (inner/outer) of $0.4 / 0.6,0.8 / 1.0$, and $1.2 / 1.4 \mathrm{~cm}$ (Figure $4 \mathrm{a}, \mathrm{b}$, and $\mathrm{c}$ respectively). The $\mathrm{CV}$ of optimum transmission is inde- pendent of the radii of the electrodes, as shown in Figure 3, with a slight decrease of CV for the $0.4 / 0.6 \mathrm{~cm}$ electrode set at the highest applied DV. The peak widths in FAIMS are a function of the applied DV as well as the radii of the electrodes. The peaks are narrow at low applied DV, for all electrode radii, but the peaks are widest for electrode set of $0.4 / 0.6 \mathrm{~cm}$ radii shown in Trace 4a. The resolution (CV/FWHM) where FWHM is the full peak width at half maximum is a function of the electrode radii. The resolution for the electrode set of $0.4 / 0.6 \mathrm{~cm}$ radii is about 3.6 to 4.5 (Figure $4 \mathrm{a}$ ), about 8.1 to 9.2 for the $0.8 / 1.0 \mathrm{~cm}$ electrode set (Figure $4 \mathrm{~b}$ ) and about 10.5 to 14 for the $1.2 / 1.4 \mathrm{~cm}$ electrodes.

Qualitatively, the width of a peak is related to the range of CV over which the ion trajectory is parallel to the walls of the electrodes. In the cylindrical geometry FAIMS, the ions migrate to, and travel parallel to the walls, at a radial distance determined by the combination of DV and CV, and the $K_{h} / K$ of the ion being studied. For a given peak in the CV scan the lowest CV of ion transmission is characterized by an optimum ion focus point located near the
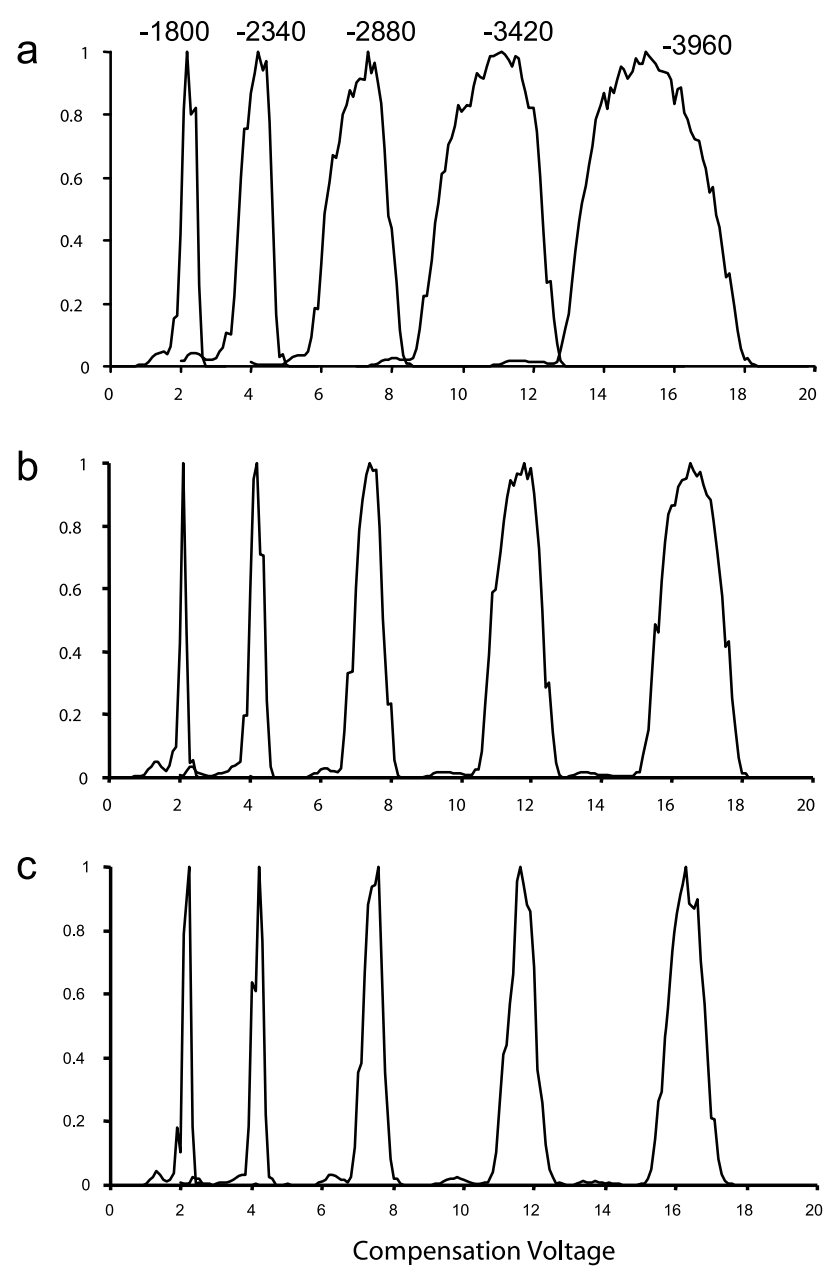

Figure 4. Individually normalized experimental CV scans acquired at DV of $-1800,-2340,-2880,-3420$, and $-3960 \mathrm{~V}$ while monitoring $\mathrm{m} / \mathrm{z}-173\left(\mathrm{BCA}^{-}\right)$using FAIMS prototypes with inner/outer electrodes of radii (a) $0.4 / 0.6 \mathrm{~cm},(\mathbf{b}) 0.8 / 1.0 \mathrm{~cm}$, and (c) $1.2 / 1.4 \mathrm{~cm}$. 


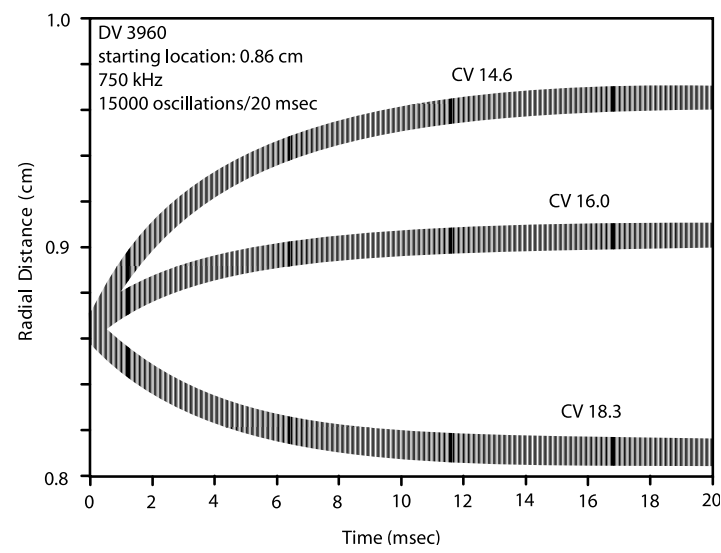

Figure 5. Calculated ion trajectories of the $m / z-173$ of $\mathrm{BCA}^{-}$, using $\alpha=7.984 \mathrm{E}-6 \mathrm{Td}^{-2}$ and $\beta=-3.049 \mathrm{E}-10 \mathrm{Td}^{-4}$ in a cylindrical FAIMS with inner/outer electrodes of radii $0.8 / 1.0 \mathrm{~cm}$. Ion trajectories were started at radius $0.86 \mathrm{~cm}$, and represent 15,000 oscillations $(20 \mathrm{~ms})$ of a $750 \mathrm{kHz}$ waveform at $\mathrm{DV}=-3960 \mathrm{~V}$ and $\mathrm{CV}=14.6,16.0$, and $18.3 \mathrm{~V}$.

outer electrode. As the CV increases to pull the ion cloud closer to the inner electrode, this focus point migrates towards the inner electrode. This is illustrated in Figure 5, which shows the ion trajectory calculations for the $\mathrm{BCA}^{-}$ anion in a FAIMS prototype with an inner electrode of radius $0.8 \mathrm{~cm}$ and outer electrode of radius $1.0 \mathrm{~cm}$ at applied DV $=-3960 \mathrm{~V}$, and CVs of 14.6, 16.0, and $18.3 \mathrm{~V}$. The trajectory is stable radially near the outer electrode at $\mathrm{CV}=14.6 \mathrm{~V}$, near the center of the annular space between the electrodes at $\mathrm{CV}=16.0 \mathrm{~V}$, and near the inner electrode near $\mathrm{CV}=18.3 \mathrm{~V}$. As a result, the peak in a CV scan might be expected to have a base width of at least $3.7 \mathrm{~V}$. Experimentally, the CV scan most appropriate for a comparison with the calculation shown in Figure 5 appears as the right-most peak shown in panel Figure $4 \mathrm{~b}$. This scan shows that the transmission of the $\mathrm{BCA}^{-}$anion can be observed from 14.9 to $17.9 \mathrm{~V}$ measured at $5 \%$ of peak height. Taken alone, in this example the ion trajectory calculation predicts a peak width about $20 \%$ wider than was observed experimentally.

Similar calculations performed using $1.2 \mathrm{~cm}$ radii inner electrode indicate that although the ion is again transmitted near $\mathrm{CV}=16.8 \mathrm{~V}$, the trajectories calculated at $\mathrm{CV}=$ 18.3 and $14.6 \mathrm{~V}$ will result in the ions colliding with the walls of FAIMS and the peak is expected to be narrower than that using the $0.8 \mathrm{~cm}$ inner electrode. Similarly, if these trajectory calculations are repeated for the $0.4 \mathrm{~cm}$ inner electrode at low DV (for example $-1800 \mathrm{~V}$ ), the optimum CV becomes low (about $2 \mathrm{~V}$ at DV $=-1800 \mathrm{~V}$ ) and the trajectory calculation predicts that the ions cannot be transmitted beyond $\mathrm{CV}$ values of $3 \mathrm{~V}$ higher or $3 \mathrm{~V}$ lower than the optimum CV. Taken alone, these simple ion trajectory calculations predict that at high DV and with narrow diameter cylinders, the transmission of $\mathrm{BCA}^{-}$will be detected over a significant range of $\mathrm{CV}$ voltages. Wide diameter FAIMS and low CV will (together and independently) result in narrow peak widths.
Qualitatively, this is consistent with the experimental data shown in Figure 4.

\section{Calculation of Peak Width in a Cylindrical Geometry FAIMS}

The ion trajectory calculation shown in Figure 5 provides an approximate prediction of the width of a peak in the CV spectrum of FAIMS. Unfortunately, the approach used in Figure 5 yields estimates of peak shapes that are much wider than are experimentally observed. A more detailed analysis based on ion mobility, ion diffusion, and ion-ion repulsion must be used to predict a peak shape that more closely resembles the experimental peak shapes shown in Figure 4.

A numerical evaluation using finite-difference methods that are commonly applied to problems in fluid dynamics was used to estimate the time-dependent behavior of the ion density between the plates of cylindrical geometry FAIMS. By analogy with fluids, the ion cloud in the FAIMS analyzer region was assumed to be a homogeneous, compressible fluid. Unlike conventional fluids, contact of this ionic "fluid" with the container walls resulted in irreversible loss of the ions by contact with the conductive electrodes of FAIMS. For these simulations: (1) the ionic cloud was subjected to diffusion which contributed to changes in density via loss at the walls of FAIMS, (2) the ionic cloud was subjected to electric fields and thus translated radially (with fields focusing the ions in some cases), and (3) the ionic cloud carried an electric charge, and therefore produced a secondary coulombic electric field which tended to disperse the cloud in the radial direction. Other sources of changes in density originating from chemical reactions and from nonuniform radial velocity profile of the carrier gas are considered later in this report.

Methods for finite-difference analysis of diffusion/ heat transfer problems [30] are readily applied to the FAIMS problem. In this report, the diffusion problem was solved using:

$$
\partial c / \partial t=D\left(\partial^{2} c / \partial r^{2}\right)
$$

which, for a finite-difference numerical simulation, is translated to

$$
c_{j}^{(i+1)}=c_{j}^{(i)}+D\left(\Delta t / \Delta r^{2}\right)\left\{\left(c_{j}^{(\mathrm{i})}-c_{j-1}^{(\mathrm{i})}\right)-\left(c_{\mathrm{j}}^{(\mathrm{i})}-c_{\mathrm{j}+1}^{(\mathrm{i})}\right)\right\}
$$

where $c$ is the ion density (concentration), $i$ is the time index, $j$ is the radial location index, $\Delta t$ (s) is the time step used in the numerical calculation, $\Delta r(\mathrm{~cm})$ is the size of radial increment used for the calculation, and $D$ is the diffusion coefficient. This equation provides an estimate of the ion density at a new time $c_{j}^{(i+1)}$ as a function of the ion density at the previous time $c_{j}^{(i)}$ and the ion density in adjacent cells radially, $c_{j-1}^{(\mathrm{i})}$ and $c_{\mathrm{j}+1}^{(\mathrm{i})}$, also from the previous time. This numerical method produces stable results within limited values of $\Delta t / \Delta r^{2}$. Figure 6 illus- 


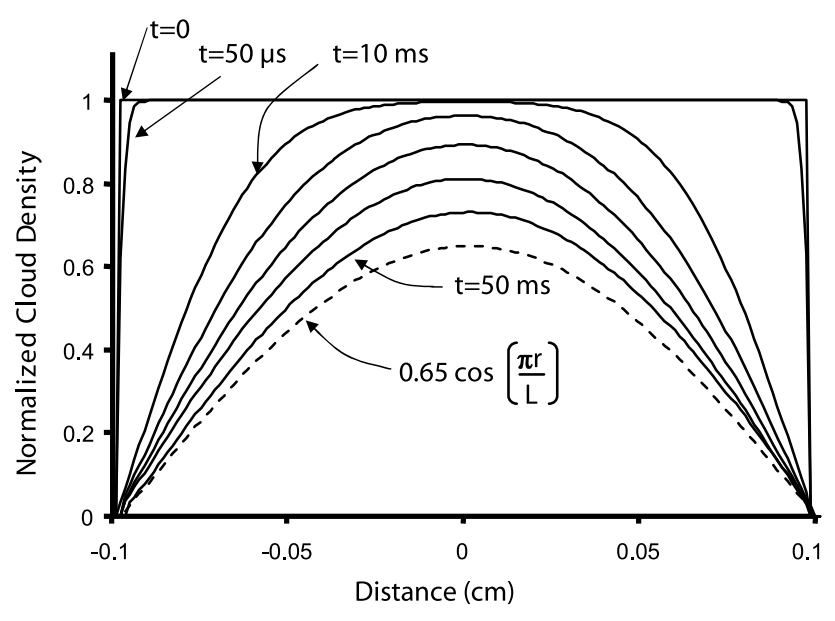

Figure 6. Calculated ion cloud density between infinite parallel conducting plates, starting with a uniform ion density of 1.0 at $\mathrm{t}=$ 0 . Ion density (solid lines) for times $t=0$ to $t=50 \mathrm{~ms}$ at $10 \mathrm{~ms}$ intervals, calculated by a finite-difference numerical method is compared with an analytical calculation [19] (dashed line) using $\mathrm{n}(r)=A \cos \left(\left[r-r_{0}\right] \pi / L\right)$ with $A=0.65, r_{0}=0$ and $L=0.2 \mathrm{~cm}$.

trates the results of a numerical simulation (solid lines) of the time-dependent redistribution of ions that were originally at uniform density in the space between two infinite parallel conductive plates.

The loss of ions from between parallel plates was evaluated analytically using the approach described by Mason and McDaniel [19]. The analytical solution for the spatial distribution of ions in a one-dimensional cavity with infinite plate walls was $\mathrm{n}(r)=A \cos \left(\left[r-r_{0}\right] \pi /\right.$ $L$ ) for the 1st mode of diffusion (higher order modes decay very rapidly), where $\mathrm{n}(r)$ is the number of particles at location $r, r_{0}$ is the center point between the plates, $A$ is the maximum number of particles, and $L$ is the distance between the plates. The result for this analytical solution is shown in Figure 6 (dotted line) with $A=0.65$ and $L=0.2$. The numerical method (solid lines) yields results in good agreement with this analytical solution. Note that the ion cloud was subject to a short period of time-dependent evolution of the radial density profile followed by a distribution whose shape was time-independent and remained as $A \cos \left(\left(r-r_{0}\right) \pi / L\right)$ for an indefinite time thereafter. All ion distributions calculated for FAIMS behave in this manner.

The ion cloud density profile shown in Figure 6 developed without external forces. If the ion cloud between two parallel conductive plates is also subjected to an electric field, the time-dependent distribution between the plates is more difficult to model analytically. However, the numerical modeling of such systems is described in most texts on calculation methods for fluid dynamics [30]. The one-dimensional array of cells representing the ion density as a function of radial distance was subjected, sequentially, to a diffusion computation (described above), and a translation computation based on a quadratic smoothing of the ion distribution followed by the calculation of the destina- tion of the distribution following the translation step. Typically, the $0.2 \mathrm{~cm}$ distance between the electrodes of FAIMS was divided into 150 to 250 radial steps, spaced to have equal areas between the steps (both flat plates and cylindrical electrodes). The sequence of diffusion/ translation was calculated as a function of time using steps of 0.01 to $0.05 \mathrm{~ms}$. This approach was tested for known situations, including the distribution described in the previous paragraph, and for translation/diffusion of packets of ions starting at a single radial location.

Figure 7 illustrates a calculation analogous to that shown in Figure 6, with an additional electric field of $5 \mathrm{~V} / \mathrm{cm}$ between the plates. The distribution of the ion cloud was calculated as a function of time for $10 \mathrm{~ms}$.
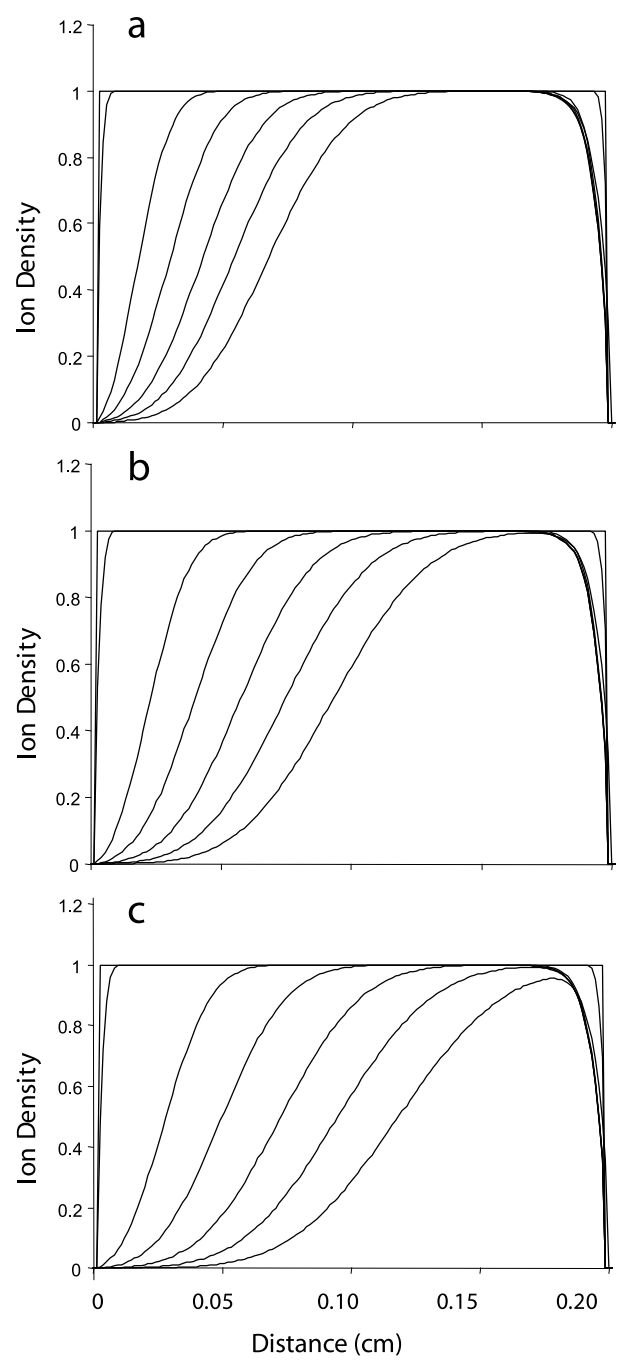

Figure 7. Calculated ion cloud density between infinite parallel conducting plates $0.2 \mathrm{~cm}$ apart with a $5 \mathrm{~V}$ difference applied to the plates. Ion densities were initially uniform, and the time-dependent evolution calculated at times $\mathrm{t}=0$ to $\mathrm{t}=10 \mathrm{~ms}$ at $2 \mathrm{~ms}$ intervals for a ions with (a) $K=1.2 \mathrm{~cm}^{2} \mathrm{~V}^{-1} \mathrm{~s}^{-1}$, (b) $\mathrm{K}=1.7$ $\mathrm{cm}^{2} \mathrm{~V}^{-1} \mathrm{~s}^{-1}$, and (c) $\mathrm{K}=2.2 \mathrm{~cm}^{2} \mathrm{~V}^{-1} \mathrm{~s}^{-1}$ using finite-difference numerical methods. In these calculations 65,51 , and $39 \%$ of the original ion density remained after $10 \mathrm{~ms}$ for each ion, respectively. 

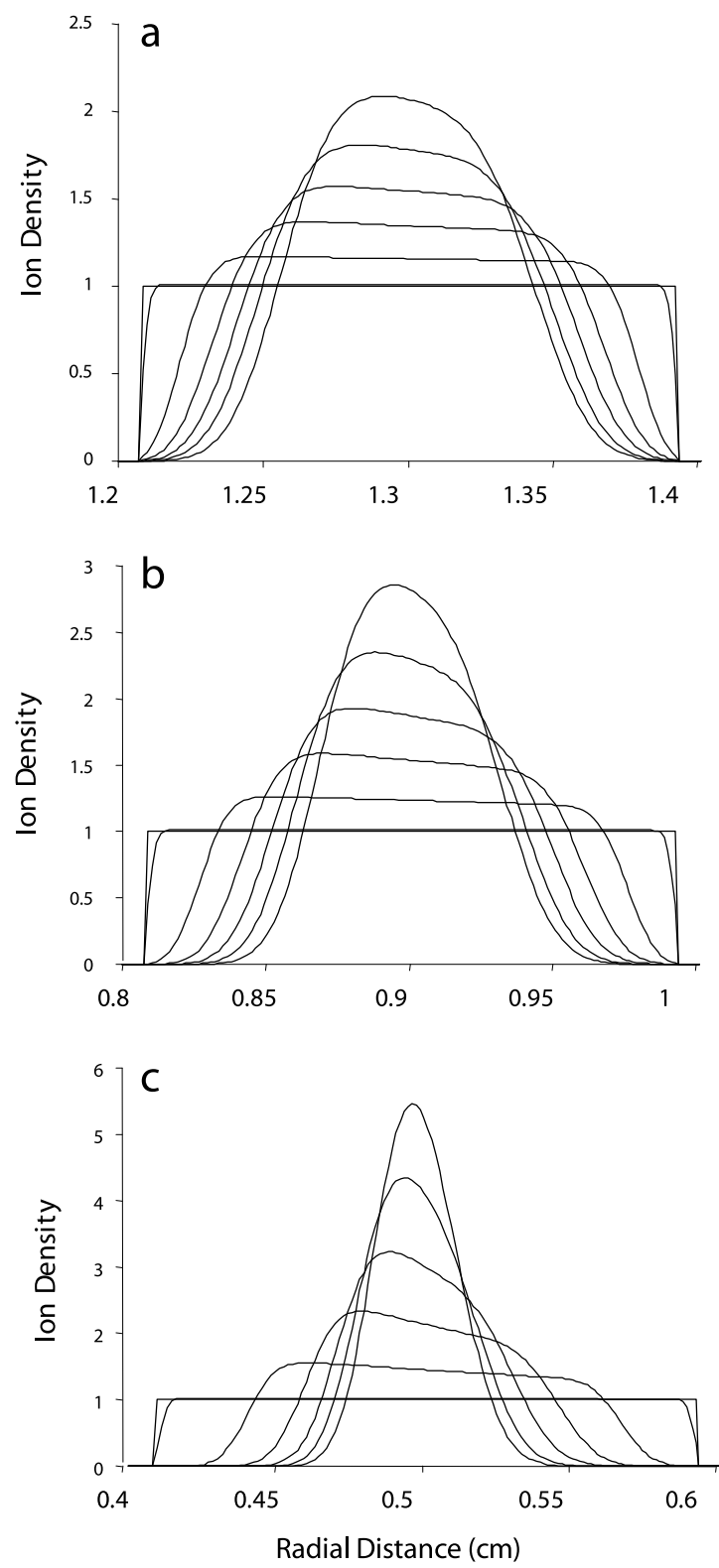

Figure 8. Calculated time-dependent evolution of the ion cloud density between cylindrical FAIMS cylinders of inner/outer electrode radii of (a) $1.2 / 1.4 \mathrm{~cm},(\mathbf{b}) 0.8 / 1.0 \mathrm{~cm}$, and (c) $0.4 / 0.6 \mathrm{~cm}$ at DV $=-4000$ and $\mathrm{CV}=16.8 \mathrm{~V}$ for an ion with $K=1.7 \mathrm{~cm}^{2} \mathrm{~V}^{-1} \mathrm{~s}^{-1}$ and $\alpha$ $=7.984 \mathrm{E}-6 \mathrm{Td}^{-2}$ and $\beta=-3.049 \mathrm{E}-10 \mathrm{Td}^{-4}$. Traces are the calculated density from $t=0$ to $t=5 \mathrm{~ms}$ at $1 \mathrm{~ms}$ intervals.

This calculation was repeated for ions having mobility (at ambient conditions) of 1.2, 1.7, and $2.2 \mathrm{~cm}^{2} \mathrm{~V}^{-1} \mathrm{~s}^{-1}$. As shown in Figure 7, the ion distribution is skewed as the ions are forced towards one of the plates, and those ions with lowest mobility move less quickly (top panel, Figure 8) towards the wall than higher mobility ions (bottom panel). After $10 \mathrm{~ms}$, the total remaining ions in the region between the plates are 65,51 , and $39 \%$ of the total ions originally occupying the space for ions with mobility $1.2,1.7$, and $2.2 \mathrm{~cm}^{2} \mathrm{~V}^{-1} \mathrm{~s}^{-1}$, respectively. Note also, that the ion cloud is not compressed under these conditions, and the ion density never exceeds the original density (taken as 1.0 in Figure 7).
The calculations of the estimated distribution of ions in a 1-dimensional space between infinite parallel plates can be extended to cylindrical geometry. The calculation of the ion density distribution in a cylindrical FAIMS is yet further complicated because of the focusing properties of FAIMS [5] illustrated by the trajectory calculations in Figure 5. The cloud of ions must be considered compressible under these conditions, and the electric fields will give rise to a convergence of the ions in radial space. This focusing field will be balanced by the dispersion action of diffusion and of ion-ion space charge repulsion.

The calculation of the virtual "focusing" fields in FAIMS has been described previously [5] but a simplified method will be described here. The behavior of the ion in strong electric fields is determined using the experimental CV, DV data described in an earlier section above. The $\alpha$ and $\beta$ values can be substituted into eq 1 to provide an instantaneous value of $\mathrm{K}_{\mathrm{h}}$ at the ambient electric field strength. The ion motion during one complete cycle of the applied waveform is calculated at each radial location between the electrodes and the magnitude of the net drift of the ion at each radial location provides the strength of the focusing action at that location. This net drift is translated into the equivalent of a dc electric field (i.e., a "virtual" electric field). The dc virtual field intensity is a function of radial location, is constant in time and is unique to the ion (via eq 1), the electrode dimensions and the CV, DV voltages applied. The time-dependent ion distribution is then calculated in the same way as described for Figures 6 and 7, in this case with the constant-in-time "focusing" virtual fields replacing the dc electric fields used in Figure 7.

Figure 8 illustrates the time-dependent simulation of the ion density of the BCA anion between concentric cylinders of FAIMS of various radii for focusing conditions of $-4000 \mathrm{~V} \mathrm{DV}$ and $16.8 \mathrm{~V} \mathrm{CV}$. The original ion density is assumed to be uniform with radial location, except that the ions cannot occupy locations close to the walls of the electrodes because of the high frequency oscillation motion due to the applied high voltage asymmetric waveform. Some ion loss due to diffusion to the walls of FAIMS is observed at short times, and the focusing fields thereafter pull the ions away from the walls. Note also, that the maximum ion density now exceeds the maximum density at $t=0$ for these conditions where the focus point lies between the cylinders of FAIMS. The ion cloud has been compressed to occupy a narrow radial band, but the total number of ions is lower than that at $t=0$, because the distribution was impinging on the walls at short times. All other parameters being equal, the time required for the ion cloud to be compressed is a function of the radii of the electrodes, as shown in Figure 8. The focusing field is strongest for narrow diameter electrodes, and weaker for the wider dimension cylindrical electrodes. The strength of the focusing field approaches zero at the infinite radii (flat plate) limit, where no ion focusing 


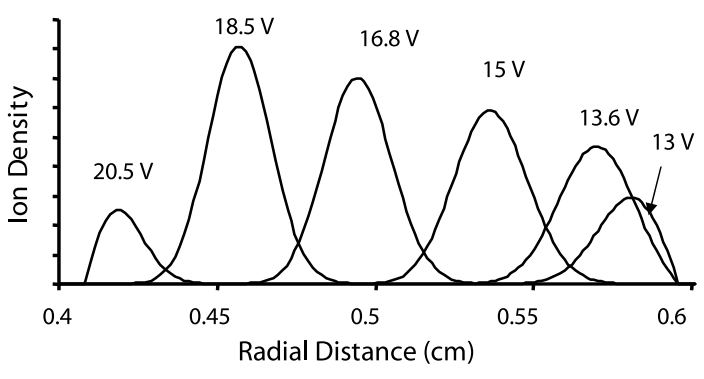

Figure 9. Calculated ion cloud densities as a function of radial distance between cylindrical FAIMS inner/outer cylinders of $0.4 / 0.6 \mathrm{~cm}$ radii after time delay of $10 \mathrm{~ms}$ with ions having $K=$ $1.7 \mathrm{~cm}^{2} \mathrm{~V}^{-1} \mathrm{~s}^{-1}, \alpha=7.984 \mathrm{E}-6 \mathrm{Td}^{-2}, \beta=-3.049 \mathrm{E}-10 \mathrm{Td}^{-4}$, at a waveform voltage of $\mathrm{DV}=-4000 \mathrm{~V}$, and at $\mathrm{CV}$ of $13,13.6,15,16.8$, 18.6, and $20.5 \mathrm{~V}$.

takes place and the ions readily disperse due to diffusion and coulombic repulsion.

The traces shown in Figure 8 were calculated at a single $\mathrm{CV}$, selected to show a condition where the ions converge to the mid-point between the electrodes. Since the radial location of the focus point is dependent on $\mathrm{CV}$ (as shown in Figure 5), ion density plots such as those shown in Figure 8 can be calculated at other values of $\mathrm{CV}$, and the ions converge to the radial location corresponding to the location of the bottom of the virtual potential well.

Figure 9 illustrates the calculated distribution of BCA anions at a DV of $-4000 \mathrm{~V}$ and at CVs of 13.0, 13.6, 15.0, $16.8,18.5$, and $20.5 \mathrm{~V}$, after $10 \mathrm{~ms}$. At time zero the ions were uniformly distributed between the electrodes of $0.4 / 0.6 \mathrm{~cm}$ inner/outer electrode radii, and the ion distribution was monitored for a period of $10 \mathrm{~ms}$. Although the time dependence of the ion density is not apparent in Figure 9, those distributions that are displaced from the electrode surfaces do not change in time because (in this simulation) no mechanism for ion loss is operative. When the ion distribution impinges on the electrode, for example at $\mathrm{DV}=-4000 \mathrm{~V}$ and $\mathrm{CV}=$ $20.5 \mathrm{~V}$ shown in Figure 9, the ion density decreases rapidly with time. In the trace at $\mathrm{CV}=20.5 \mathrm{~V}$, approximately $19 \%$ of the original ion density remains after $10 \mathrm{~ms}$. For the calculations at CV 18.5, 16.8, and $15.0 \mathrm{~V}$, approximately $98 \%$ of the original ion density remains. The intensity distributions vary in height because the strength of ion focusing varies with radial distance. From Figure 9 it is clear that a peak in the CV scan is actually a series of measurements in which the optimum focus radial position is moved from close to the outer electrode (at lower $\mathrm{CV}$ ) to nearer the inner electrode (higher $\mathrm{CV}$ ). Ions are lost to the walls extremely rapidly when the focus location is (in theory) inside the wall of either electrode, less rapidly if the focus location is between the electrodes but is near the wall of either electrode, and minimally if the focus point falls midway between the electrodes. The ion distributions calculated in Figure 9 agree well with experimentally observed ion density profiles between the electrodes of cylindrical FAIMS [5].
Using the methods described above, the timedependent distributions of the ions can be calculated for any condition of DV and CV. This residual ion density, after a selected time delay, is calculated at a series of $C V$ values at constant $D V$, to generate a calculated CV scan. Figure 10a shows a simulated CV scan for a $0.8 / 1.0 \mathrm{~cm}$ electrode set at DV $-3960 \mathrm{~V}$ where the traces show the residual ion density at $50 \mathrm{~ms}$ intervals up to to $250 \mathrm{~ms}$. At short residence times in the FAIMS analyzer the peaks are expected to be wider than the peaks at longer residence times. For $\mathrm{BCA}^{-}$at the applied CV and DV, the peak width will be invariant for residence times in the FAIMS analyzer longer than about $250 \mathrm{~ms}$. Figure $10 \mathrm{~b}$ is a repeat of the calculations of Figure 10a, but with the addition of the effect of coulombic ion-ion repulsion. The ion-ion repulsion acts in a direction to expand the radial dimensions of the ion clouds shown in Figures 8 and 9, and as a result the ion cloud is more likely to be in contact with the electrodes and ion density decreases with time. In Figure 10a the calculated boundaries of the ion cloud are not in direct contact with an electrode between the $C V$ values of 15.5 and about $17.5 \mathrm{~V}$, and the calculation predicts no loss of ions. Any mechanism that broadens the ion cloud, including enhanced diffusion, coulombic repulsion, or chemical reactions, will widen the radial distribution of the ions and narrow the $\mathrm{CV}$ peak via collisions with the electrode surfaces.
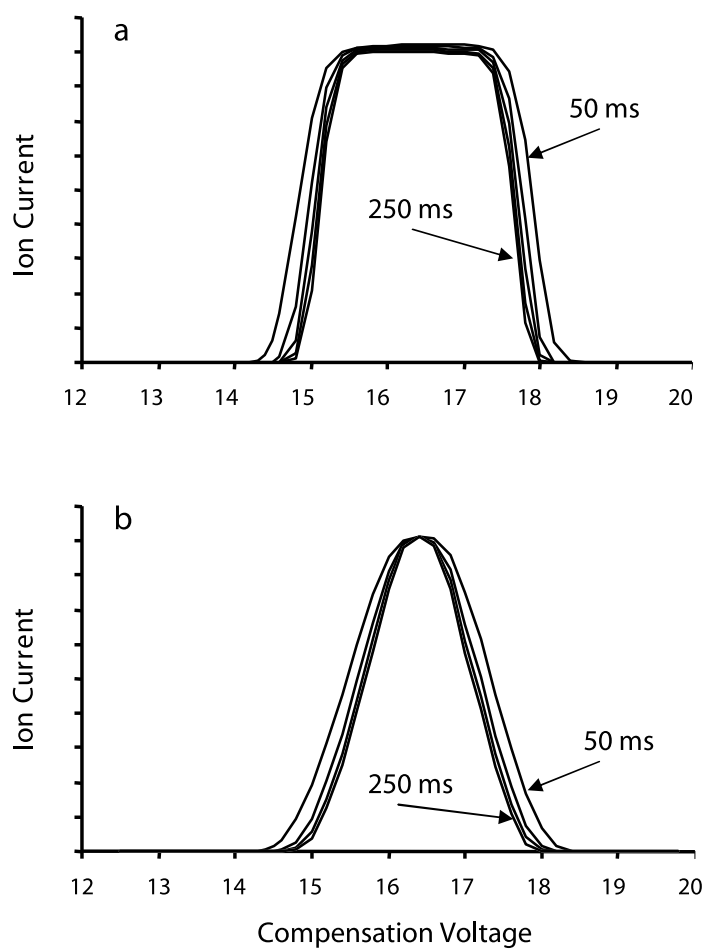

Figure 10. Calculated $\mathrm{CV}$ peak shapes based on residence times of 50 to $250 \mathrm{~ms}$ in a $0.8 / 1.0 \mathrm{~cm}$ radii cylindrical FAIMS. The ion cloud density was calculated at $50 \mathrm{~ms}$ intervals with ions having $K=1.7 \mathrm{~cm}^{2} \mathrm{~V}^{-1} \mathrm{~s}^{-1}, \alpha=7.984 \mathrm{E}-6 \mathrm{Td}^{-2}, \beta=-3.049 \mathrm{E}-10 \mathrm{Td}^{-4}$, at $\mathrm{DV}=-3960 \mathrm{~V}$ and $\mathrm{CV}$ from 12.6 to $19.8 \mathrm{~V}$ in $0.1 \mathrm{~V}$ increments. Calculated (a) without and (b) with coulombic ion-ion repulsion. 

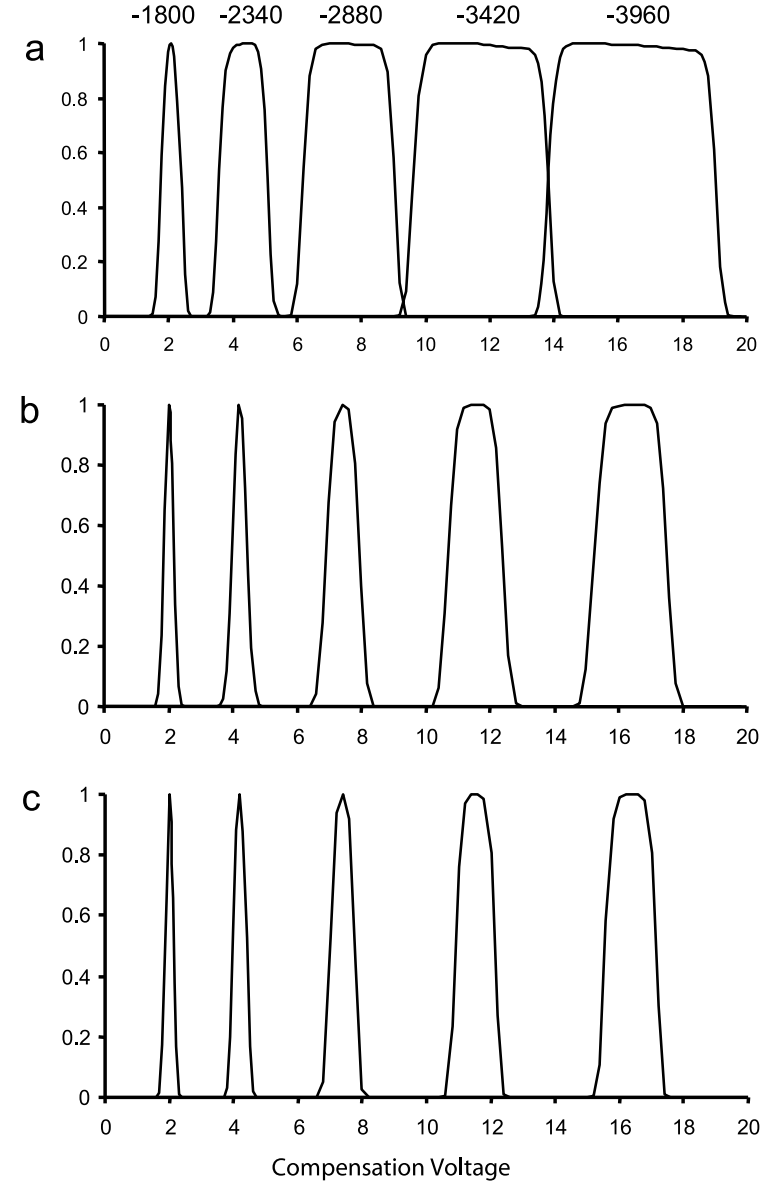

Figure 11. Calculated CV peak shapes for FAIMS with cylinders inner/outer radii (a) $0.4 / 0.6 \mathrm{~cm},(\mathbf{b}) 0.8 / 1.0 \mathrm{~cm}$, and (c) $1.2 / 1.4 \mathrm{~cm}$ at DV of $-1800,-2340,-2880,-3420$, and $-3960 \mathrm{~V}$, using $K=1.7$ $\mathrm{cm}^{2} \mathrm{~V}^{-1} \mathrm{~s}^{-1}, \alpha=7.984 \mathrm{E}-6 \mathrm{Td}^{-2}, \beta=-3.049 \mathrm{E}-10 \mathrm{Td}^{-4}$. At each CV the ion cloud was initially uniform and the evolution of the density profile for a $250 \mathrm{~ms}$ period was calculated. Coulombic repulsion was ignored.

Figure $10 \mathrm{~b}$ indicates that the radial distribution of the ions has been widened sufficiently that the ions will be lost to the electrodes at voltages between 15.5 and $17.5 \mathrm{~V}$. The peak shape appears triangular when coulombic repulsion within the ion cloud is considered in these calculations. The minimum ion loss occurs when the virtual potential well falls approximately mid-way between the electrode surfaces.

\section{Comparison of Experimental, Calculated Peak Widths in Cylindrical Geometry FAIMS}

Figure 4 illustrated the normalized experimental peaks collected for $\mathrm{BCA}^{-}$in cylindrical geometry FAIMS prototypes with inner/outer electrodes of radii 0.4/0.6, $0.8 / 1.0$, and $1.2 / 1.4 \mathrm{~cm}$. For comparison, Figure 11 illustrates the normalized peaks resulting from the simulation of the time-dependent ion density within the FAIMS analyzer for the same conditions of DV and CV, using an ion residence time of $250 \mathrm{~ms}$ in FAIMS. Based on the traces shown in Figure 10, the calculated peak shape is not expected to change when the calculation is extended beyond $250 \mathrm{~ms}$, even if the ion residence time in the experimental apparatus is significantly longer than $250 \mathrm{~ms}$. In contrast, however, other ion loss mechanisms discussed below, including mechanical and chemical processes, continue for the total duration of the ion residence time in the apparatus and the calculation cannot be truncated prematurely at $250 \mathrm{~ms}$. The calculations used to produce the traces in Figure 11 were based upon the effect of ion focusing and diffusion on the ion cloud, whereas the effects of ion-ion mutual electrostatic repulsion and random ion redistributions were neglected in this calculation.

The traces for the simulation in the $0.4 / 0.6 \mathrm{~cm}$ FAIMS, shown in the top panel in Figure 11, indicate that the peak is expected to have a flat top at high applied DV. This is predicted qualitatively by the ion trajectory calculations shown in Figure 5 and by the ion density modeling shown in Figure 9. For the small radius cylindrical FAIMS operated at high DV, the focus region remains between the cylinders of FAIMS for several volts of applied CV, and therefore the peak is expected to be very wide. Wide peaks were experimentally observed (Figure 4a) for the $0.4 / 0.6 \mathrm{~cm}$ FAIMS, but the peaks did not have a uniformly flat top shape.

The traces in Figure 4 and Figure 11 are normalized, and relative intensity information is not apparent. The data in Figure 2a was collected using electrodes with $0.8 / 1.0 \mathrm{~cm}$ radii and illustrates the changes in relative intensity observed experimentally as the applied waveform voltage is increased. Figure $4 \mathrm{~b}(0.8 / 1.0 \mathrm{~cm}$ radii electrodes) and Figure 2 share several of the same data traces. For comparison, the relative intensity of the five traces in Figure 4b (experimental results) were 9, 11, 29, 62 , and 100 for applied DV $-1800,-2340,-2880$, -3420 , and -3960 , respectively, whereas the relative calculated intensities in Figure 11b were 40, 83, 98, 100, and 100 for the same applied DV values.

Several assumptions were implicit in the calculation leading to Figure 11. First, it was assumed that the diffusion coefficient was invariant with $\mathrm{E} / \mathrm{N}$; second, it was assumed that the ion-ion electrostatic repulsive interactions in the cloud was negligible; and finally, it was assumed that mechanical (gas turbulence) and chemical ion redistribution played no role in ion distribution between the electrodes.

\section{Refinements to Calculated Peak Shapes in Cylindrical Geometry FAIMS}

Figure 11 illustrates the shapes of expected peaks in FAIMS based on the physics of the ion focusing in the virtual electric fields in FAIMS and the counterbalancing effects of diffusion. The square topped peaks shown in Figure 11 are seldom seen experimentally, and have only been routinely observed for those ions having extremely strong focusing (very high CV) in FAIMS. 
This suggests that the calculations leading to the peak shapes shown in Figure 11 underestimate some mechanism of ion scattering, or redistribution, in the analyzer region of FAIMS. Sources of such additional widening of the ion cloud density profile are considered below.

Diffusion at high E/N. The diffusion coefficient is a function of E/N [19]. The effective temperature experienced by an ion drifting in a strong electric field is described by $T_{\text {eff }}=T+M v_{d}^{2} / 3 k$ where $T$ is the ambient gas temperature, $M$ is the molar mass of the bath gas, $v_{d}$ is the ion velocity, and $k$ is Boltzmann's constant. Since the velocity of the ion is $K E$ where $K$ is the ion mobility and $E$ is the electric field, the diffusion coefficient varies with the strength of the applied electric field.

If a waveform with $\mathrm{DV}=-4000 \mathrm{~V}$ is applied to FAIMS with electrodes spaced $0.2 \mathrm{~cm}$ apart, the peak electric field corresponds to about $20,000 \mathrm{~V} / \mathrm{cm}$. The $\mathrm{BCA}^{-}$anion, with a mobility (296 K and 760 torr) of about $1.7 \mathrm{~cm}^{2} \mathrm{~V}^{-1} \mathrm{sec}^{-1}$, will experience an effective temperature of about $425 \mathrm{~K}$ during the application of an electric field of $20,000 \mathrm{~V} / \mathrm{cm}$ in 760 torr nitrogen. Using the estimation that $D=k T_{\text {eff }} K / q$, where $D$ is the diffusion coefficient, $k$ is Boltzmann's constant, $T_{\text {eff }}$ is the effective temperature, $K$ is the ion mobility, and $q$ is the charge on the ion, the diffusion coefficient of an ion at an effective temperature of about $430 \mathrm{~K}$ is about $0.063 \mathrm{~cm}^{2} \mathrm{~s}^{-1}$. Under the same conditions, absent the electric field, the diffusion coefficient would be $0.043 \mathrm{~cm}^{2} \mathrm{~s}^{-1}$. The effective diffusion of the $\mathrm{BCA}^{-}$anion increases by about $50 \%$ in a field of $20,000 \mathrm{~V} / \mathrm{cm}$. This represents the maximum instantaneous increase in the effective diffusion that can be achieved under the experimental conditions discussed in this report.

During the application of the asymmetric waveform, the ion spends very little time at the maximum electric field, thus a more realistic estimate of the average effective temperature can be calculated using the average ion velocity during one cycle of the waveform. Using this approach, the diffusion coefficient is about $0.048 \mathrm{~cm}^{2} \mathrm{~s}^{-1}$.

The diffusion of an ion moving in a strong field has a directional component. $D$ is usually resolved into $D_{L}$ and $D_{T}$, the longitudinal and transverse coefficients of diffusion (where the directions are taken relative to the direction of the electric field). Although the detailed calculations leading to $D_{L}$ and $D_{T}$ can be complex, an estimate of these can be obtained from directional estimates of the effective temperature:

$$
\begin{aligned}
& k T_{L}=k T+\frac{1}{3}\left(\frac{4 m+M}{2 m+M}\right) M \nu_{d}^{2} \\
& k T_{T}=k T+\frac{1}{3}\left(\frac{m+M}{2 m+M}\right) M \nu_{d}^{2}
\end{aligned}
$$

where $T_{L}$ and $T_{T}$ are the effective temperatures in the longitudinal and transverse directions relative to the field, $T$ is the ambient gas temperature, $M$ is the molar mass of the bath gas, $m$ is the molar mass of the ion, $v_{d}$ is the ion velocity, and $k$ is Boltzmann's constant. The estimates of effective temperature are subsequently used to estimate the coefficient of diffusion in the longitudinal and transverse directions using:

$$
\begin{aligned}
& D_{L}=\left(k T_{L} / q\right) K\left(1+\frac{d \ln K}{d \ln E}\right) \\
& D_{T}=\left(k T_{T} / q\right) K
\end{aligned}
$$

where $q$ is the charge on the ion, $K$ is the ion mobility, and $E$ is the electric field.

Assuming the term in the right-most brackets in eq 8 is close to 1 , the coefficient of diffusion in the longitudinal direction, when applying the waveform described above, is about $0.052 \mathrm{~cm}^{2} \mathrm{~s}^{-1}$. Using this estimated value of the augmented diffusion coefficient (in the radial direction in the case of FAIMS), the calculation of the peak shapes in the CV scans for the three FAIMS prototypes was repeated. The changes in the peak shapes were small because the magnitude of the increase in diffusion resulting from the application of a waveform up to DV $=-4000 \mathrm{~V}$ did not significantly widen the profile of the ion distribution in these FAIMS devices. Therefore, the increased effective temperature experienced by the ion, and the concomitant increase in ionic diffusion are not the main contributors to differences between the calculated (Figure 11, calculated without field-augmented diffusion), and experimental (Figure 4) peak shapes. Shvartsburg et al. [17] have also concluded that the variation of the coefficient of diffusion in strong electric fields does not materially affect peak shapes in FAIMS. A further discussion of the effective temperature experienced by the ion appears in a later section.

Coulombic ion-ion repulsion. Space charge can readily be incorporated into a finite-difference numerical modeling of the ion cloud. For this report, the electric field generated by a cloud of ions was estimated and was superimposed upon the virtual electric field originating from the ion focusing effect in FAIMS. Gauss's law can be used to show that the uniform electric field outside of an infinite sheet of charge density $\rho$ is $\rho / 2 \varepsilon$ where $\varepsilon$ is the dielectric constant of the medium surrounding the sheet. If this sheet of charge is located at the center point between two infinite conductive surfaces, both electrically grounded, the electric field remains $\rho / 2 \varepsilon$ because an opposite and equal electric charge is induced in the surfaces of the conductive plates. The cloud of ions (having a nonuniform radial distribution) between the plates of FAIMS was divided into a series of narrow uniform sheets of charge, and the electric field on either side of the sheet calculated. In planar geometry the location of the sheet of charge between the plates and the ion density in the sheet are used to calculate the 


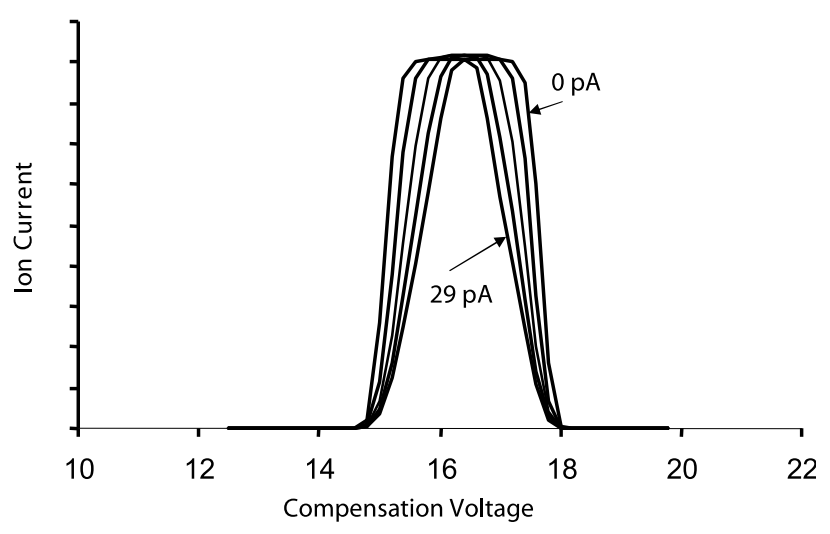

Figure 12. Calculated CV peak shapes for FAIMS with inner/ outer cylinders $0.8 / 1.0 \mathrm{~cm}$, for an ion with $K=1.7 \mathrm{~cm}^{2} \mathrm{~V}^{-1} \mathrm{~s}^{-1}, \alpha$ $=7.984 \mathrm{E}-6 \mathrm{Td}^{-2}$, and $\beta=-3.049 \mathrm{E}-10 \mathrm{Td}^{-4}$, with $\mathrm{DV}=-3960 \mathrm{~V}$. The ion cloud density was calculated at $250 \mathrm{~ms}$ for CV from 12.6 to $19.8 \mathrm{~V}$ at increments of $0.2 \mathrm{~V}$, and the residual ion density plotted as a percentage of the total ion density at $t=0$. The space charge ion-ion repulsion was calculated assuming charge density corresponding to $0,7.3,14.6,21.9$, and $29 \mathrm{pA}$ current passing through the electrode at a gas flow of $0.5 \mathrm{~L} / \mathrm{min}$.

uniform electric field generated on either side of this sheet. In cylindrical geometry the space charge induced field must be calculated for sheets of ions at each radial location, and for each sheet at all radial locations on both sides of the sheet of charge density. The sum of the contributions from each such sheet provides an estimate of the field at a given radial location. At each radial location this space charge induced electric field was superimposed on the virtual focusing electric field at that radial location. Since the ion cloud was mobile (and compressible) in the electric fields, the space charge induced fields were recalculated at each point in time.

Figure 12 illustrates the effect of ion-ion space charge on the shape of the calculated CV peak for the $0.8 / 1.0 \mathrm{~cm}$ radius FAIMS at DV $=-3960 \mathrm{~V}$ using several estimations of ion density in the annular analyzer region. At the start of the time-dependent calculation of the profile of the ion density in FAIMS the electric current was assumed to be $0,0.9,1.9,2.8$, and $3.7 \times 10^{-12} \mathrm{coul} / \mathrm{cm}^{3}$ evenly distributed between the electrodes. Assuming a flow of carrier gas through FAIMS of approximately $500 \mathrm{~mL} / \mathrm{min}$, an ion current of about $29 \mathrm{pA}$ would be flowing through FAIMS at the highest space charge used in Figure 12.

The electric fields introduced by space charge act in the same direction as diffusion and widen the radial distribution of ions between the electrodes. This reduces the voltage range over which the ions are effectively transmitted through FAIMS. As shown in Figure 12 , the peaks in the CV spectrum will be somewhat more triangular in shape as a result of the added effect of ion-ion repulsion. However, the width of the peak at its base is constant because the ion density approaches zero and space charge becomes negligible under conditions used to calculate the ion density for those $\mathrm{CV}$ values near the foot of the CV peak. Although it has been proposed that peak shape in FAIMS is strongly influenced by ion-ion repulsion [17], for the experiments reported in this manuscript good agreement between calculated and experimental peak shape can be produced without space charge repulsion.

Figure 13 illustrates the calculated peak shapes for $\mathrm{BCA}^{-}$using the three different radii of FAIMS electrodes. The space charge effect has been ignored. The calculation used to generate the $\mathrm{CV}$ traces shown in Figure 13 began by assuming the cloud of ions was initially distributed evenly between the electrodes. The ion cloud was allowed to redistribute under the influence of diffusion and the virtual potential well in FAIMS, much as shown in Figure 8. However, unlike the calculations shown in Figure 11, the cloud was not allowed to develop for a full $250 \mathrm{~ms}$, but rather was allowed to develop for only $24 \mathrm{~ms}$. It was then assumed that the ion cloud was (mechanically or chemically) forced to return to its original uniform distribution in
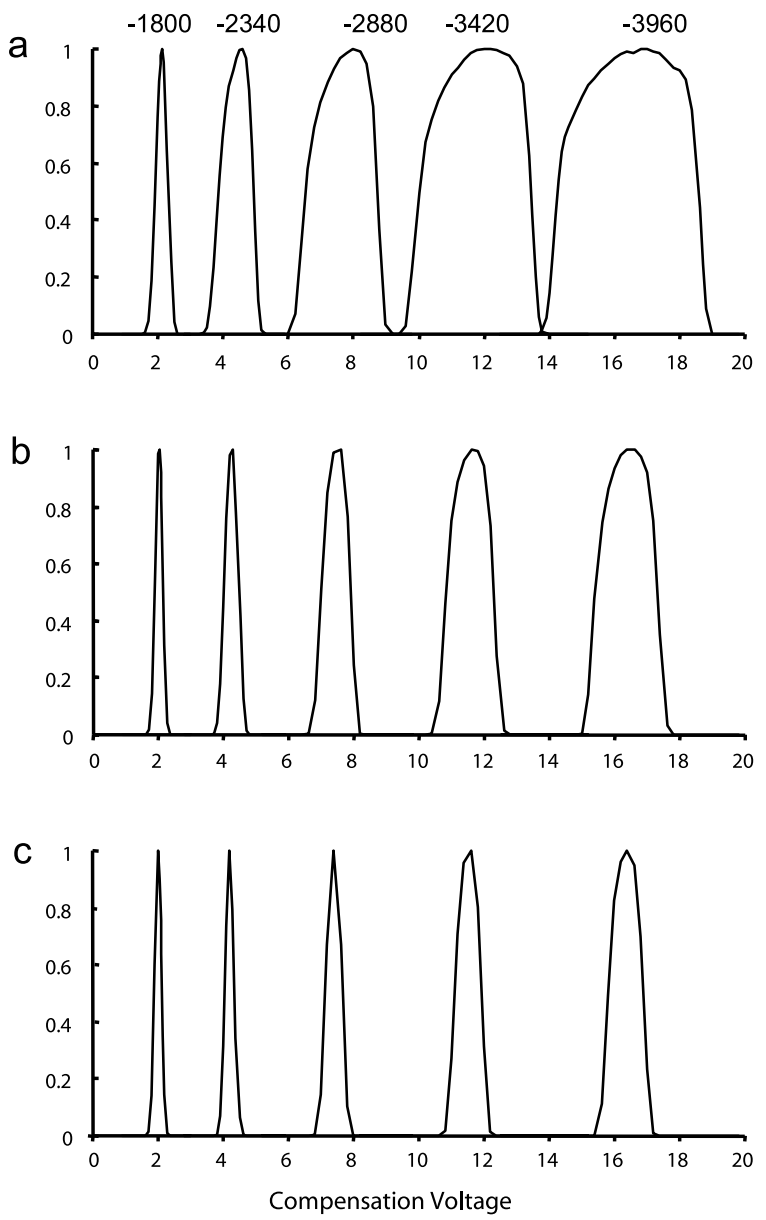

Figure 13. Calculated CV peak shapes for FAIMS with cylinders of inner/outer radii (a) $0.4 / 0.6 \mathrm{~cm}$, (b) $0.8 / 1.0 \mathrm{~cm}$, and (c) $1.2 / 1.4$ $\mathrm{cm}$ at DV of $-1800,-2340,-2880,-3420$, and $-3960 \mathrm{~V}$, using $K$ $=1.7 \mathrm{~cm}^{2} \mathrm{~V}^{-1} \mathrm{~s}^{-1}, \alpha=7.984 \mathrm{E}-6 \mathrm{Td}^{-2}, \beta=-3.049 \mathrm{E}-10 \mathrm{Td}^{-4}$. Coulombic repulsion was ignored. At each $\mathrm{CV}$ the ion cloud was initially uniform, followed by $24 \mathrm{~ms}$ evolution of the density profile. The cloud was forced back to uniform after each $24 \mathrm{~ms}$, in a cyclic process repeated 30 times. 
the radial direction and allowed to develop for another $24 \mathrm{~ms}$ period. This process was repeated 30 times to approximate ion transport time of about $900 \mathrm{~ms}$. In contrast to the calculation that was used to produce Figure 11, which gives invariant results for any time beyond $250 \mathrm{~ms}$, a calculation that involves random ion loss is operative over the entire ion residence time and cannot be truncated as early as $250 \mathrm{~ms}$. Reasonable agreement between the experimental data and the calculations appeared to suggest long ion residence times.

The peak shapes calculated by this method, shown in Figure 13, are in excellent agreement with the experimentally measured peaks shown in Figure 4. In particular, the skewed shaped peaks collected at DV -2340 and -3420 for the $0.4 / 0.6 \mathrm{~cm}$ radii, appear to be matched very well by these calculations. The data in Figure 4 and Figure 13 have been normalized, but to assist in comparison between these figures, the relative intensities of the five experimental peaks in Figure $4 \mathrm{~b}$ $(0.8 / 1.0 \mathrm{~cm}$ electrodes) were 9, 11, 29, 62, and 100 for applied DV $-1800,-2340,-2880,-3420$, and -3960 , respectively, and the relative calculated peak heights shown in Figure $13 \mathrm{~b}(0.8 / 1.0 \mathrm{~cm}$ electrodes) were 0.6, $19,58,85$, and 100 respectively. However, at DV $-3960 \mathrm{~V}$ the peak shapes and absolute CV values for the 0.4 / $0.6 \mathrm{~cm}$ radii electrodes do not match the experimental peak shapes as well as for the lower DV values, and for the wider electrodes. Further methods of refinement of the calculation of the peak shape in FAIMS are considered below.

\section{Other Considerations for Calculated Peak Widths in Cylindrical Geometry FAIMS}

Radial gas velocity profiles at low Reynolds numbers. At a gas flow of about $0.5 \mathrm{~L} / \mathrm{min}$ through concentric FAIMS inner/outer electrodes with radii of $0.8 / 1.0 \mathrm{~cm}$, the calculated average linear velocity $v_{g}$ of the gas flow is about $7 \mathrm{~cm} / \mathrm{s}$. Taking the distance parameter $\mathrm{L}$ to be the spacing between the electrodes of $0.2 \mathrm{~cm}$, the gas viscosity $\eta$ of $1.83 \times 10^{-4}$ poise and the gas density $\rho_{g}$ of $1.25 \times 10-3 \mathrm{gm} / \mathrm{cc}$, the dimensionless Reynolds number (Re) is $\rho_{g} v_{g} \mathrm{~L} / \eta$, equal to about 10 . The flow is expected to be turbulent when Re exceeds 2000 .

The modeling of ion density profiles described in this report assumes that the cloud of ions travels in a medium having no velocity profile in the radial direction. In fact, at low Reynolds numbers, the gas velocity profile in the radial direction will assume the usual parabolic shape, with the gas velocity in the center of the profile traveling approximately twice as fast as the average flow. The parabolic shape will be slightly modified by the cylindrical geometry of FAIMS, especially for small radii electrodes. The nonuniform gas velocity profile is expected to distort the radial ion density profile. Nevertheless, this parabolic flow profile is not expected to materially change the peak shapes calculated in Figure 11 because no new ion loss mechanism is introduced via this velocity profile.

Gas flows that are not laminar would have a major effect on the ion density profile between the FAIMS electrodes. The focusing electric fields in FAIMS are weak, and any bulk mixing or systematic swirls in the gas flows will significantly alter the ionic distribution in the FAIMS analyzer. Several sources of gas mixing are possible, including (1) turbulence originating at the ion inlet that subsides gradually, (2) rotating swirl formed as the gas follows a curved path around the electrode, and (3) gas rotation and/or cyclone that may occur near the gas outlet near the hemispherical terminus of the inner electrode. The calculated CV traces in Figure 13 more closely match the experimental curves in Figure 4 than did the calculated curves in Figure 11, and appear to support the proposal that the ion cloud is subject to mechanical and/or chemical redistribution during transit through the FAIMS electrodes.

Ion loss via chemical reactions. An ion loss mechanism related to gas-phase ion molecule reactions has been discussed previously [5, 31]. It is assumed that new complexes will form between an ion and polar contaminant species (including $\mathrm{H}_{2} \mathrm{O}, \mathrm{CO}_{2}$, etc.) in the carrier gas. In FAIMS, because of the application of the high voltage asymmetric waveform, the ions are subjected to strong fields (and elevated effective temperatures) on an intermittent basis. This means that the complexes will tend to dissociate during the high voltage portion of the waveform, and will be short lived if the gas-phase concentration of these polar contaminants is low. Nevertheless, some complexes will form, and will exist for periods beyond the length of a single cycle of the applied asymmetric waveform. During a scan of CV, the focus point of an ion shifts from near the outer wall (lower CV) to the inner wall (higher CV). When the ion-neutral complex (of multi-cycle lifetime) is formed it will migrate in a direction dependent on the $\mathrm{K}_{\mathrm{h}} / \mathrm{K}$ ratio of this newly formed ion. If the ion-neutral complex is formed near a wall, and if the DV and CV combination cause the complex to migrate towards this nearby wall, the ion will be lost. If the same complex is formed while the focus point is located adjacent to the opposite wall, the ion-neutral species will probably dissociate before traversing the FAIMS analyzer region, and the ion will return to the optimum focus point. This chemical redistribution of ions in the FAIMS analyzer may operate with effects similar to that of mechanical redistribution via gas turbulence described above. Again, the model used to generate Figure 13 suggests that one (or more) mechanisms of redistribution of the ions are operative in FAIMS.

Imperfections in the physical geometry of FAIMS. Although it is assumed throughout this report that the mechanical components of FAIMS are fabricated exactly, the FAIMS prototype is subject to two types of mechanical error. Ideally, first, the inner electrode is aligned paral- 
lel to the center axis of the outer electrode, and, second, the center axis of the inner electrode is located at the center axis of the outer electrode. Evaluation of the effects of mechanical errors on peak width and intensity would be required prior to establishing the mechanical tolerances for construction of these devices.

A further, important, mechanical factor influences peak shapes in the type of design of FAIMS reported here. With the present design, the inner electrode is manually translated longitudinally inside the FAIMS, to establish the optimum distance between the terminus of the inner electrode, and the end of the outer electrode. Since the behavior of the ion cloud between the electrodes of FAIMS is highly dependent on the strength of the electric fields produced by the applied DV and CV voltages, the change from a two-dimensional cylindrical geometry, to a three-dimensional spherical geometry at the terminus of the inner electrode, is expected to modify the ion density profile. For the data collected for this report, the inner electrode was adjusted to a longitudinal position that did not affect the peak shapes in the CV scan. However, it is known that the adjustment of the longitudinal position of the inner hemispherical tipped electrode is critical to the peak shapes, sensitivity, and resolution of domed inner electrode FAIMS devices, and is a subject of ongoing investigations.

\section{Conclusions}

The peak shape of the CV spectrum has been evaluated for dome type cylindrical geometry FAIMS electrodes with inner electrode radii of $0.4,0.8$, and $1.2 \mathrm{~cm}$ with a $0.2 \mathrm{~cm}$ wide analyzer region. The peaks in the $\mathrm{CV}$ spectrum are narrow at low applied waveform voltages, and widen as the applied voltage is increased. At a given applied waveform voltage, the peak shapes are narrow for electrodes having wider diameter electrodes. The qualitative changes in peak widths over a range of applied DV and electrode radii are readily predicted using simple ion trajectory calculations. Peak shapes that closely match experimental data can be calculated using fluid dynamics methods with incorporation of ion focusing, ion diffusion, and redistribution of ion density by mechanical processes.

\section{Acknowledgments}

The authors acknowledge the contribution of Applied Biosystems/MDS-Sciex who provided equipment for this work. They thank D. A. Barnett and B. Ells for the help provided during this research and M. Guevremont for her help during the preparation of this manuscript.

\section{References}

1. Gorshkov, M. P. The method of impurity analysis in gases. USSR Inventors certificate no. 966583; 1982.
2. Carnahan, B. L.; Tarassov, A. S. Ion mobility spectrometer. US Patent 5420424; 1995.

3. Buryakov, I. A.; Krylov, E. V.; Nazarov, E. G.; Rasulev, U. K. A new method of separation of multi-atomic ions by mobility at atmospheric pressure using a high-frequency amplitudeasymmetric strong electric field. Int. J. Mass Spectrom. Ion Processes 1993, 128, 143-148.

4. Purves, R. W.; Guevremont, R.; Day, S.; Pipich, C. W.; Matyjaszczyk, M. S. Mass spectrometric characterization of a high-field asymmetric waveform ion mobility spectrometer. Rev. Sci. Instrum. 1998, 69, 4094-4105.

5. Guevremont, R.; Purves, R. W. Atmospheric pressure ion focusing in a high-field asymmetric waveform ion mobility spectrometer. Rev. Sci. Instrum. 1999, 70, 1370-1383.

6. Guevremont, R.; Purves, R. W.; Barnett, D. A.; Ding, L. Iontrapping at atmospheric pressure (760 torr) and room temperature with a high-field asymmetric waveform ion mobility spectrometer (FAIMS). Int J. Mass Spectrom. 1999, 193, 45-56.

7. Guevremont, R.; Ding, L.; Ells, B.; Barnett, D. A.; Purves, R. W. Atmospheric pressure ion trapping in a tandem FAIMS-FAIMS coupled to a TOFMS: Studies with electrospray generated gramicidin S ions. J. Am. Soc. Mass Spectrom. 2001, 12, 1320-1330.

8. Guevremont, R.; Ding, L.; Purves, R. W.; Barnett, D. A. Atmospheric pressure trapping of amino acids using a FAIMS ion trap coupled to a quadrupole/time-of-flight mass spectrometer. Proceedings of the 50th ASMS Conference on Mass Spectrometry and Allied Topics; Orlando, FL, June, 2002.

9. Ells, B.; Barnett, D. A.; Purves, R. W.; Guevremont, R. Detection of nine chlorinated and brominated haloacetic acids at part-per-trillion levels using ESI-FAIMS-MS. Anal. Chem. 2000, 72, 4555-4559.

10. Ells, B.; Barnett, D. A.; Purves, R. W.; Guevremont, R. Trace level determination of perchlorate in water matrices and human urine using ESI-FAIMS-MS. J. Environ. Monitor. 2000, 2, 393-397.

11. Guevremont, R.; Barnett, D. A.; Purves, R. W.; Vandermey, J. Analysis of a tryptic digest of pig hemoglobin using ESIFAIMS-MS. Anal. Chem. 2000, 72, 4577-4584.

12. McCooeye, M. A.; Ells, B.; Barnett, D. A.; Purves, R. W.; Guevremont, R. Quantitation of morphine and codeine in human urine using high-field asymmetric waveform ion mobility spectrometry (FAIMS) with mass spectrometric detection. J. Anal. Toxicol. 2001, 25, 81-87.

13. McCooeye, M. A.; Mester, Z.; Ells, B.; Barnett, D. A.; Purves, R. W.; Guevremont, R. Quantitation of amphetamine, methamphetamine, and their methylenedioxy derivatives in urine by solid-phase microextraction coupled with electrospray ionization-high field asymmetric waveform ion mobility spectrometry-mass spectrometry. Anal. Chem. 2002, 74, 3071-3075.

14. Purves, R. W.; Barnett, D. A.; Ells, B.; Guevremont, R. Gasphase conformers of the $[\mathrm{M}+2 \mathrm{H}] 2+$ ion of bradykinin investigated by combining high-field asymmetric waveform ion mobility spectrometry, hydrogen/deuterium exchange, and energy-loss measurements. Rapid Commun. Mass Spectrom. 2001, 15, 1453-1456.

15. Purves, R. W.; Ding, L.; Ells, B.; Guevremont, R.; Barnett, D. A. Detection of protein variants using ESI-FAIMS-MS. Proceedings of the 50th ASMS Conference on Mass Spectrometry and Allied Topics; Orlando, FL, June, 2002.

16. Borysik, A. J. H.; Read, P.; Little, D. R.; Bateman, R. H.; Radford, S. E.; Ashcroft, A. E. Separation of $\beta 2$-microglobulin conformers by high-field asymmetric waveform ion mobility spectrometry (FAIMS) coupled to electrospray ionization mass spectrometry. Rapid Commun. Mass Spectrom. 2004, 18, $2229-2234$. 
17. Shvartsburg, A.; Tang, K.; Smith, R. D. Modeling the resolution and sensitivity of FAIMS analyses. J. Am. Soc. Mass Spectrom. 2004, 15, 1487.

18. Shvartsburg, A.; Tang, K.; Smith, R. D. Understanding and designing FAIMS separations in gas mixtures. Anal. Chem., 2004, 76, 7366-7374.

19. Mason, E. A.; McDaniel, E. W. Transport properties of ions in gases; John Wiley and Sons, Inc.: New York, 1988; pp 173-178

20. Rorrer, L. C., III; Guevremont, R.; Barnett, D. A.; Yost, R. A. The role of clustering on ion behavior in FAIMS. Proceedings of the 50th ASMS Conference on Mass Spectrometry and Allied Topics; Orlando, FL, June, 2002.

21. Eiceman, G. A.; Krylov, E.; Krylova, N.; Nazarov, E. G.; Miller, R. A. Separation of ions from explosives in differential mobility spectrometry by vapor-modified drift gas. Anal. Chem. 2004, 76, 4937-4944.

22. Shvartsburg, A.; Tang, K.; Smith, R. D. Optimization of the design and operation of FAIMS analyzers, unpublished.

23. Venne, K.; Bonneil, E.; Eng, K.; Thibault, P. Enhanced sensitivity in proteomics analyses using NanoLC-MS and FAIMS. PharmaGenomics 2004, 4.

24. Guevremont, R.; Barnett, D. A.; Purves, R. W.; Viehland, L. A. Calculation of ion mobilities from electrospray ionization high-field asymmetric waveform ion mobility mass spectrometry data. J. Chem. Phys. 2001, 114, 10270-10277.

25. Viehland, L. A.; Guevremont, R.; Purves, R. W.; Barnett, D. A. Comparison of high-field ion mobility obtained from drift tubes and a FAIMS apparatus. Int. J. Mass Spectrom. 2000, 197, 123-130.

26. Guevremont, R. FAIMS: A new tool for mass spectrometry. J. Chromatogr. A, in press.

27. Handy, R.; Barnett, D. A.; Purves, R. W.; Horlick, G.; Guevremont, R. Determination of nanomolar levels of perchlorate in water by ESI-FAIMS-MS. J. Anal. At. Spectrom. 2000, 15, 907-911.

28. Barnett, D. A.; Ells, B.; Guevremont, R.; Purves, R. W.; Viehland, L. A. Evaluation of carrier gases for use in high-field asymmetric waveform ion mobility spectrometry (FAIMS). J. Am. Soc. Mass Spectrom. 2000, 11, 1125-1133.

29. Guevremont, R. http://www.faims.com

30. Abbott, M. B.; Basco, D. R. Computational Fluid Dynamics; Longman Scientific \& Technical; New York: Wiley, 1989.

31. Barnett, D. A.; Purves, R. W.; Ells, B.; Guevremont, R. Separation of ortho-, meta-, and para-phthalic acids by high-field asymmetric wavefrom ion mobility spectrometry using mixed carrier gases. J. Mass Spectrom. 2000, 35, 976-980. 